\title{
An evolutionary framework for studying mechanisms of social behavior
}

\author{
NESCent Working Group on Integrative Models of Vertebrate Sociality: Evolution, \\ Mechanisms, and Emergent Properties, Hans A. Hofmann ${ }^{1}$, Annaliese K. Beery ${ }^{2}$, \\ Daniel T. Blumstein ${ }^{3}$, Iain D. Couzin ${ }^{4}$, Ryan L. Earley ${ }^{5}$, Loren D. Hayes ${ }^{6}$, Peter L. Hurd ${ }^{7}$, \\ Eileen A. Lacey ${ }^{8}$, Steven M. Phelps ${ }^{1}$, Nancy G. Solomon ${ }^{9}$, Michael Taborsky ${ }^{10}$, \\ Larry J. Young ${ }^{11}$, and Dustin R. Rubenstein ${ }^{12}$
}

\footnotetext{
${ }^{1}$ The University of Texas at Austin, Department of Integrative Biology and Institute for Cellular and Molecular Biology, 2415 Speedway, Austin, TX 78712, USA

${ }^{2}$ Smith College, Department of Psychology and Program in Neuroscience, Northampton, MA 01063, USA

${ }^{3}$ University of California, Department of Ecology and Evolutionary Biology, 621 Young Drive South, Los Angeles, CA 90095-1606, USA

${ }^{4}$ Princeton University, Department of Ecology and Evolutionary Biology, Princeton, NJ 08644, USA

${ }^{5}$ University of Alabama, Department of Biological Sciences, 300 Hackberry Lane, Box 870344, Tuscaloosa, AL 35487, USA

${ }^{6}$ University of Tennessee at Chattanooga, Department of Biological and Environmental Sciences, Chattanooga, TN 37403, USA

${ }^{7}$ University of Alberta, Department of Psychology and Centre for Neuroscience, Edmonton, Alberta, T6G 2E9, Canada

${ }^{8}$ University of California at Berkeley, Museum of Vertebrate Zoology and Department of Integrative Biology,

3101 Valley Life Sciences Building, Berkeley, CA 94720-3160, USA

${ }^{9}$ Miami University, Department of Biology, Oxford, OH 45056, USA

${ }^{10}$ University of Bern, Institute of Ecology and Evolution, Division of Behavioural Ecology, Wohlenstrasse 50a, 3032 Hinterkappelen, Switzerland

${ }^{11}$ Emory University, Center for Translational Social Neuroscience, Yerkes National Primate Research Center, 954 Gatewood Road, Atlanta, GA 30329, USA

${ }^{12}$ Columbia University, Department of Ecology, Evolution and Environmental Biology, 1200 Amsterdam Avenue, New York, NY 10027, USA
}

\begin{abstract}
Social interactions are central to most animals and have a fundamental impact upon the phenotype of an individual. Social behavior (social interactions among conspecifics) represents a central challenge to the integration of the functional and mechanistic bases of complex behavior. Traditionally, studies of proximate and ultimate elements of social behavior have been conducted by distinct groups of researchers, with little communication across perceived disciplinary boundaries. However, recent technological advances, coupled with increased recognition of the substantial variation in mechanisms underlying social interactions, should compel investigators from divergent disciplines to pursue more integrative analyses of social behavior. We propose an integrative conceptual framework intended to guide researchers towards a comprehensive understanding of the evolution and maintenance of mechanisms governing variation in sociality.
\end{abstract}

Corresponding authors: Hofmann, H.A. (hans@utexas.edu); Rubenstein, D.R. (dr2497@columbia.edu).

Keywords: evolution; social behavior; complex sociality; group-living; neural circuits; hormones; genomics.
The study of social behavior in the 21st century

All animals interact with conspecifics at some point in their lives. Members of the same species tend to be each other's fiercest competitors and strongest allies, as evidenced by the intense cooperation and conflict that characterize many intraspecific interactions [1]. These interactions are the products of genetic, epigenetic, endocrine, and neural mechanisms that - in conjunction with environmental conditions - affect Darwinian fitness and evolve via natural selection. Building upon Aristotle's four questions, Tinbergen [2] posited that understanding behavior requires the integration of studies of mechanism and function. Only by asking questions both from a proximate perspective (i.e., focusing on causation and development) and an ultimate perspective (i.e., focusing on adaptive value and evolutionary descent) can behavior be fully understood. Social behavior in particular lends itself to such an integrative approach not only because it commands the attention of many disciplines [3] but also because even many behaviors commonly considered nonsocial often occur in a social context (e.g., mating, fighting, parental care). Social behavior is also special because the selective agents are other members of the same species, and this results in intriguing evolutionary dynamics. Nevertheless, in the intervening decades since Tinbergen's 
seminal work [2] studies of behavioral mechanisms have proceeded largely independently of analyses of ultimatelevel explanations for social behavior [4]. Among the factors contributing to this disconnect are the challenges of applying laboratory methods to field research where most complex social behaviors are studied, as well as longstanding differences in terminology, conceptual foci, and study taxa [3,5-7]. Progress towards an integrated understanding of the evolution of social behavior has been limited.

Only now, 50 years after Tinbergen's seminal 1963 publication [8], efforts to integrate neural, genetic, epigenetic, physiological, ecological, and evolutionary studies of behavior are gaining increased prominence [7,9-11,101], facilitated by multiple factors, including innovative technologies (e.g., high-throughput sequencing [12]), and analytical procedures (e.g., improved statistical methods for modeling and comparative analyses [13]) as well as the increasing ease of application of these advances to field studies (e.g., biotelemetry $[14,15])$. As a result, it is increasingly possible to address all four of Tinbergen's questions concurrently for the same species $[3,7,10,11,16]$, which is most effective when using modern comparative methods [13]. Such integration is crucial if studies of behavior are to contribute to solutions to pressing biological problems. For example, only by understanding the evolutionary origins of diverse mechanisms can we begin to predict how species will respond to global change [17]. Similarly, a thorough understanding of the adaptive consequences of diverse mechanisms can help to identify novel model systems for studies of specific neuropsychiatric disorders [18]. Integrating Tinbergian levels of analysis is especially appropriate for the study of social behavior which, given its complexity, must be approached from an integrative perspective.

\section{Historical perspectives}

Although most current textbooks on animal behavior prominently feature Tinbergen's four questions [19-21], researchers have been slower to adopt the type of truly integrative approach that Tinbergen originally proposed [2]. Indeed, studies of behavior remain to some extent divided into efforts to understand ultimate- versus proximate-level reasons for variation in social interactions [3]. Each tradition offers important impulses for the integrative conceptual framework we outline below.

\section{Ecological and evolutionary traditions}

Ethologists and behavioral ecologists have emphasized field studies of ultimate-level aspects of social behavior. Crucial concepts addressed by such studies include the roles of kinship and inclusive fitness in shaping social interactions, as well as the effects of specific ecological parameters on social structure [8,22]. Such studies have the advantage of documenting patterns of behavior and the associated adaptive consequences in the environments, and under the selective regimes experienced by the study organisms. However, such analyses have tended to ignore the physiological, neural, and genetic mechanisms underlying these behavioral patterns as part of a 'phenotypic gambit', a heuristic construct positing that detailed knowledge of the mechanistic bases for behavior is not required for an understanding of its function and evolution $[23,24]$. As a result, such studies have been typically unable to determine how underlying mechanisms shape observed behavioral responses to external environments, including generating significant individual variation in response to similar external environments.

\section{Neuroendocrine and genetic foundations}

Psychologists and neuroscientists interested in social behavior have followed an often parallel but distinct research tradition that emphasizes its physiological, neuroendocrine, and genetic bases. Prominent themes have included the roles of learning and ontogenetic experience on social interactions, as well as the effects of hormone levels in both generating and mediating specific patterns of behavior. Such studies are typically conducted under laboratory conditions and involve a limited number of 'model' study organisms, thereby offering important opportunities for controlled experimentation, often employing tools specific to the organisms under study. However, these analyses have tended to employ highly inbred study organisms that live in simplistic laboratory environments [25], thereby largely precluding consideration of the functional contexts in which behavior - particularly complex social behavior - occurs and has evolved [26]. As a result, studies of proximate-level mechanisms of social behavior generally cannot address the potential impacts of variable environmental conditions.

\section{The power of integration}

Although numerous opportunities exist for multidisciplinary research, at present we lack an appropriate conceptual framework - including a common language for describing social behavior - to develop an integrative understanding of the evolution of social behavior. To capitalize upon emerging opportunities we need predictive models of social interactions that integrate function and mechanism, and that can be applied to diverse taxa over a range of social and ecological contexts. We offer here such an integrative framework of sociality (Figure 1A), one that incorporates individual variations in ecology, fitness, and experience as well as the neural, physiological, genetic, and developmental mechanisms underlying social behavior. We outline ways in which researchers can use this framework to dissect mechanisms of social behavior in free-living animals exposed to the real-world ecological and evolutionary factors that shape such behavior. We do so in a manner that will open up innovative avenues for comparison across disparate taxonomic groups. Importantly, this framework can be extended to other types of complex behaviors (e.g., finding food or a suitable habitat, migratory behavior, learning and memory formation) and therefore acts as a blueprint for the integrative study of behavior.

\section{An integrative framework}

Clearly, combining proximate and ultimate approaches to the same phenomenon generates opportunities for understanding social behavior that are not possible through either tradition alone. For example, because the genetic, molecular, and neural mechanisms underlying behavior are subject to selection and have a phylogenetic 


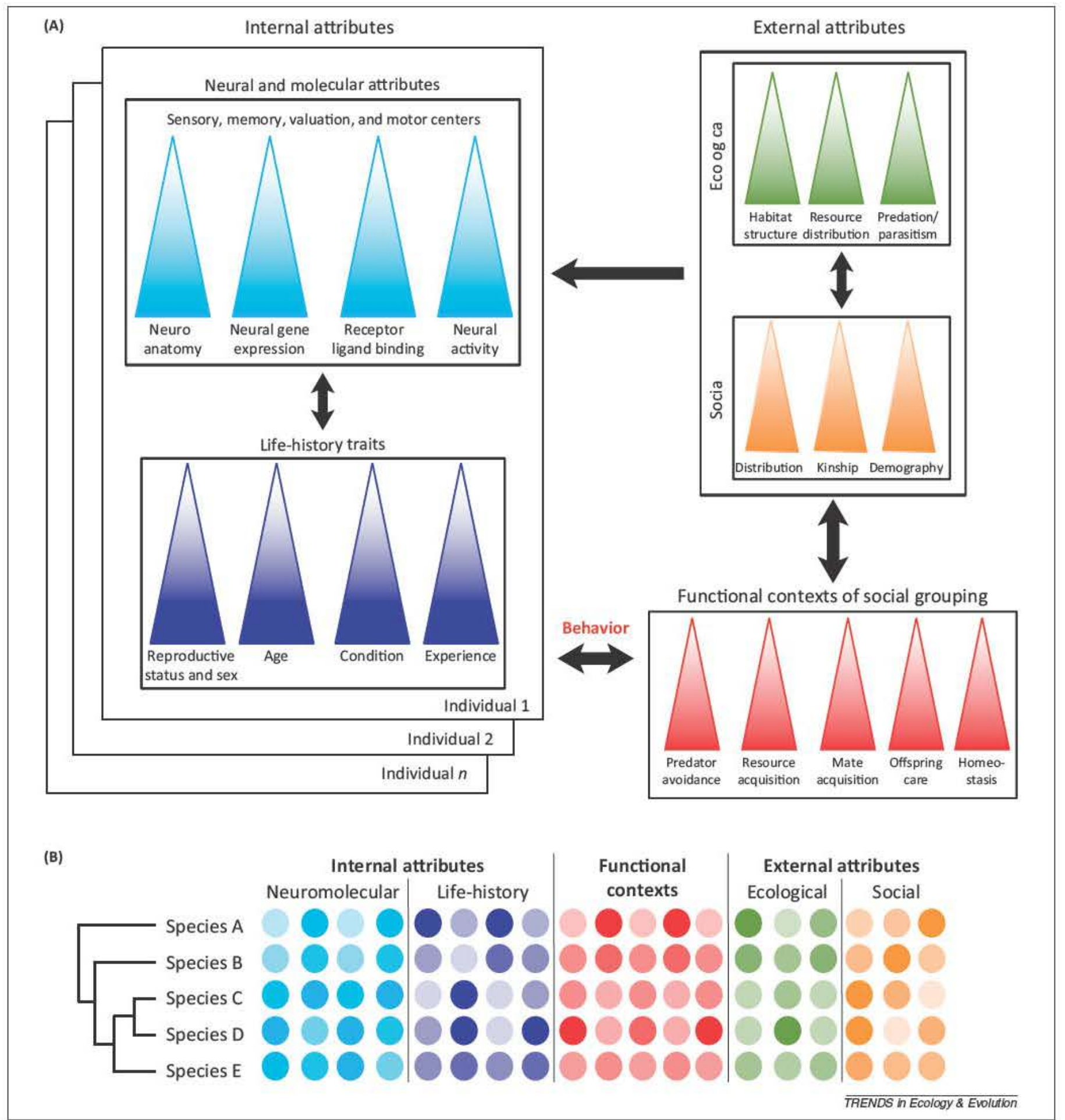

Figure 1. An integrative framework for the study of social behavior. (A) The framework incorporates external (ecology and social environment) and internal attributes (neural and molecular measures together with intrinsic life-history traits) in which individuals and populations can vary. Note that even subtle differences over time and among individuals or species in neural/molecular characteristics can result in functional variation, giving rise to behavioral diversity. Triangles with gradients represent the continuous or semi-continuous nature of the variables, indicating a range from high to low. (B) Evolutionary processes influence internal attributes (such as neural and molecular mechanisms and life-history traits) in relation to external attributes (ecological characteristics and social group traits) and determine social behavior within different functional contexts. Multivariate approaches can be used to identify co-variance patterns within and across populations or species at ecological, individual, social, and/or mechanistic levels. Variation in color intensity represents quantitative variation in the attributes similar to the gradients in the triangles in (A).

history, they need to be understood in a variety of social and ecological contexts within an explicitly integrative framework [1]. Conversely, understanding the nature of these mechanisms can help to reveal why responses to variable environmental conditions take the forms that they do [27]. The study of social behavior, in particular, is uniquely positioned to benefit from such integration for several reasons. First, as noted above, social behavior is 
nearly ubiquitous, with clear functional ties to crucial issues such as conservation and human health. Second, previous research has been remarkably productive in identifying the ecological conditions that shape the social behavior of a wide range of taxa (e.g., [9,28,29]). Third, detailed investigations of the mechanistic bases for social behavior have been completed for several model organisms (e.g., [30,31]), providing an important baseline for studies of other taxa. Although studies of social behavior are not unique in offering such opportunities, few aspects of organismal biology are as clearly and firmly poised to forge innovative and integrative perspectives on phenotypic variation [32].

Developing a truly integrative view of social behavior requires an appropriate conceptual framework that will (i) facilitate identification of general, potentially causal, relationships between behavior and other aspects of the biology of an organism, (ii) improve our ability to generate testable predictions regarding these relationships, and (iii) enhance our ability to identify the most suitable study systems for a given behavioral attribute. We propose here such a framework that is aimed at (i) facilitating understanding of the diversity of regulatory processes of social behavior in an ecological and evolutionary context, (ii) providing a roadmap for generating testable predictions from existing data, and (iii) identifying suitable model systems for simultaneous study in the laboratory and field. We believe that this framework serves to bridge the historical conceptual gap between relevant biological disciplines, thereby paving the way for a comprehensive and truly integrated understanding of social behavior.

\section{Functional explanations for proximate mechanisms Causes for social grouping}

Social behavior occurs in many forms and contexts, but group-living organisms exhibit some of the most complex forms of social behavior. Understanding how and why animals form groups represents an ideal situation in which to develop an integrative framework of complex social behavior because it involves many forms of positive and negative social interactions. Empirical studies of vertebrates and invertebrates have demonstrated that animals typically form groups for one or more of five functional reasons: (i) predator avoidance; (ii) resource acquisition; (iii) mate acquisition; (iv) offspring care; and (v) homeostasis [33] (Figure 1A). These functional contexts, however, cannot always be clearly distinguished when conspecifics interact under natural conditions. Understanding the mechanisms underlying social grouping can provide insights into why groups form and, perhaps more importantly, why group-living has evolved. For example, we are only beginning to understand that the neural and molecular mechanisms underlying social behaviors - as is the case for all phenotypes - are the result of interactions between genetic, environmental, developmental, and epigenetic processes [7]. Comparative studies have illuminated the behavioral, neural, and molecular underpinnings of social behavior, suggesting that mechanisms regulating behavior in similar contexts might, in part, be highly conserved across diverse vertebrate taxa, as has been suggested for paternal care in mammals and teleost fishes [34]. By contrast, similar behaviors in different contexts or timeperiods can also result from different mechanisms. For example, territorial aggression, in the context of mate acquisition, is often modulated by sex steroids, such as androgens, whereas aggression outside of reproduction is often modulated by other hormonal mechanisms [35]. Importantly, temporal differences in neurochemical and molecular regulatory mechanisms or variation across individuals or species result in functional variation in sensory, memory, valuation, and motor centers. Thus, the expression of seemingly identical behavioral patterns in different reproductive contexts or seasons - or in different individuals or species - might involve diverse regulatory processes. Understanding these processes in the context of social behavior can help to inform us how and when groups form, and whether similar associations in different species are driven by the same or different underlying mechanisms.

\section{Neural mechanisms in social species}

Modern biology has long moved beyond the fruitless debate about the relative contributions of nature versus nurture, and instead has come to the insight that behavior - in common with any other phenotype - is the result of interactions among genetic, environmental, developmental, and epigenetic processes. Nevertheless, how these neural and molecular mechanisms evolve is much less well understood. Four different hypotheses have been proposed [7,36]: (i) the neural and molecular substrates of behavior might be conserved even though the resulting behavior patterns have evolved in parallel (deep homology [37]); (ii) independently evolved mechanisms might result in similar behavioral functions (e.g., [38]); (iii) molecular and neural pathways might diverge through time with no concomitant change in the phenotype (developmental system shift [39]); or (iv) conserved molecular mechanisms can become associated with divergent functions and phenotypes over evolutionary time (phenologs [40]). These apparently opposing scenarios are in fact not mutually exclusive, and all can shape different behavioral phenotypes across populations or species such that a given functionally equivalent behavioral phenotype might arise from several different mechanisms.

Support can be found for all four hypotheses in a diversity of social organisms. For example, monogamous mating systems have evolved independently numerous times in many taxa, but the formation of pair bonds might involve different (e.g., prairie vole vs California mouse [38]) or conserved (e.g., prairie voles and convict cichlid fish $[30,41])$ neuroendocrine pathways. Similarly, there is significant neuroendocrine variation in the regulation of territorial aggression, but the central role of the biogenic amine serotonin appears to be conserved across animals [42-44]. A well-known example of developmental system drift (i.e., developmental pathways diverge in response to selection, although the resulting phenotypes do not change) concerns sex-determining mechanisms, where very different underlying mechanisms involving chromosome dosage, sex-determining genes, or environmental factors such as temperature or social status [45-47] give rise to males and females with sex-specific behaviors. In 
the context of social behavior, developmental system drift can mean that behavioral responses or brain regions that regulate behavior can be homologous even though their morphological substrates or developmental origins are not [7]. Phenologs, by contrast, comprise conserved gene networks which become associated with very different phenotypes over the course of evolution [40]. For example, nonapeptides regulate pair-bonding behaviors across vertebrates [30,41], and orthologs of the oxytocin or vasopressin ancestral gene also regulate mating behavior in nematodes [48] and leeches [49]. The study of these convergent and divergent pathways in conjunction with a detailed understanding of the survival value and fitness consequences of specific behavior patterns promises to yield insights into general principles underlying social evolution at both proximate and ultimate levels.

\section{Conceptual relationships between mechanisms and function}

A comprehensive understanding of variation in sociality requires not only the study of social behavior (i.e., the interactions among conspecifics) but also of reproductive behavior (i.e., the regulation of who mates with whom) and social organization (i.e., the patterns of association within and between groups) (see [22] for detailed discussion). Moreover, a truly integrative understanding of social evolution requires the reconstruction of the evolutionary histories of social traits and the characterization of relationships among the different regulatory mechanisms responsible for patterns of social behavior. Distinct behavioral traits do not operate independently and are not acted upon by selection in isolation from one another, even though they are usually studied in this manner [26]. Instead, suites of behavioral patterns commonly co-vary, forming overall social systems and life-history strategies that can differ within and among individuals [50], as well as across populations and species [51]. Similarly, behavioral patterns generally co-vary with endocrine and neural measures. For example, across vertebrates, competing phenotypes often differ in trade-offs between traits that affect fitness, including body coloration, aggression, and immune function [52,53]. Strong correlational selection is generally thought to result in such coadapted trait complexes [54], with pleiotropic hormonal systems playing a central role $[55,56]$. Neuroendocrine systems might thus promote or constrain divergence and speciation because the effects of disruptive selection on one trait are transferred to the other trait in either a synergistic or antagonistic manner [53,56].

We propose a framework for the integrative study of complex social behavior that formalizes conceptual relationships between mechanisms and function (Box 1). Specifically, we propose a list of attributes, either external (e.g., ecological characteristics or social and/or demographic traits of the group) or internal (i.e., neural and molecular characteristics, life-history traits) that can be quantified (repeatedly and simultaneously, if necessary) in multiplyinteracting individuals (Figure 1A). Importantly, these attributes are much broader than the kinds of elemental behavior patterns (e.g., aggression towards an intruder; dichotomous female mate-choice) that are typically examined in most mechanistic studies conducted in laboratory

\section{Box 1. An integrative framework of sociality}

Our framework explains patterns of social behavior that are most frequently studied (e.g., mating behavior, offspring care). In reality, these apparently disparate behavioral patterns are linked by ecological factors at one causal level and a common neuromole cular substrate at another. Thus, both ultimate and proximate forces will shape and constrain behavioral strategies to vary along principal component dimensions. Similarly, there are functional relationships between individual neural and molecular attributes (e.g., hormone levels are functionally linked to receptor densities). Components of variation in these dimensions will reflect the organization of parts of the mechanism into a functioning whole. For example, behavioral patterns cluster into functional sets (e.g., monogamous pair bonding, parental care, territorial defense, etc.). Each principal component of variation in traits such as neurotrans mitter and neuromodulator expression and reception in the nuclei of the social decision making network in these organisms should relate to biologically meaningful variation in behavior. A reasonable starting point is to model a one to one correspondence between the principal components of behavior and those of the mechanistic underpinnings. Aside from this larger aim of identifying correlations between axes of mechanisms and axes of behavior, there is a practical benefit to analyzing principal components of behavioral variation, or variation in mechanism: to identify the set of the most robust, efficient, proxy measures for causal mechanisms and/or behavioral variation.

settings. We also propose a multivariate approach for identifying patterns of covariance and for reducing complexity in such datasets (e.g., principal components at ecological, individual, social, and mechanistic levels) with the goal of unraveling the processes that govern the evolution of the neural and molecular mechanisms underlying social behavior (Figure 1B). These insights provide quantifiable variables that can facilitate a thorough understanding of, and generate testable predictions on, the causes, origins, and functional consequences of behavioral variation within and across populations and species.

\section{External attributes: ecological characteristics and social group traits}

The mechanisms regulating social behaviors are affected by external conditions including the ecology and social environment of an individual (Figure 1A). Importantly, these attributes can differentially affect group members. For example, habitat structure, resource distribution, or risk of predation and parasitism, can differentially influence the behavior of dominant and subordinate, or male and female, group mates [57]. Such parameters can also influence the distribution and behavior of one sex, which in turn can affect the behavior of the opposite sex [58]. Likewise, the demographic and kin composition of a population can affect decision-making in juveniles (Box 2) [5961]. The costs and benefits of living in groups can affect the evolution of neural pathways underlying aggressive and cooperative behaviors, which in turn might affect group composition and persistence, and ultimately population structure (e.g., estrildid finches [62]).

\section{Internal attributes: life-history traits}

The neural processes underlying social behaviors are also influenced by a variety of attributes of the individual, including sex, reproductive state, age, condition, and experience (Figure 1A), all of which can affect the opportunities 
Social behavior entails both negative (e.g., aversive, aggressive) and positive (e.g., affiliative, cooperative) interactions. Negative interac tions often lead to dispersal (i.e., emigration from natal group) which has been well studied in numerous species and ecological contexts. Although some hormonal and physiological factors have been identified that relate to dispersal (e.g., glucocorticoid levels, organiza tional hormonal factors, body condition [94 96]), little is known about the underlying neural circuits. Integrative approaches to dispersal might include neuroendocrine profiling before and after dispersal from the natal group, comparisons between dispersers and non dispersers within a population, and other temporal changes in group structure such as immigration that result in seasonal group forma tion.

Studies of affiliative behavior, by contrast, have already begun to integrate neurobiological and ecological data. In prairie voles, dopamine, oxytocin, and vasopressin act within the mesolimbic reward pathway to establish pair bonds between mates [30]. Across Microtus vole species, differences in the distribution of oxytocin and vasopressin receptors as well as estrogen receptor $\alpha$ are associated with species differences in mating strategy [97,98], and studies that investigate the consequences of these variations in field settings have been conducted $[99,100]$. Work in seasonally social meadow voles as well as in colonial South American rodents has suggested parallel and potentially convergent pathways by which oxytocin receptor density is involved in natural variation in affiliative behavior and group living outside the context of monogamy [78]. In estrildid finches, homologous peptide receptors modulate group size pre ferences and are differentially distributed in species that exhibit year round territoriality or flocking, and the peptide neurons that supply these receptors respond selectively to positive social stimuli [62]. These species share ecological and other aspects of social organiza tion (e.g., all are monogamous and biparental), thus a major question is how the mechanisms of pair bonding, gregariousness, and territoriality evolve in other taxa where the ecological, social, and functional contexts can be different. In prairie voles, for example, vasopressin receptor expression in the cingulate cortex predicts reproductive success specifically in wanderers, but not in pair bonded males [99], indicating that space use patterns need to be considered to understand individual variation in receptor expres sion. To understand behavioral diversity fully we must place these, and additional studies, into a broad ecological and evolutionary context. of an individual during competition to access resources such as mates and breeding sites. Moreover, within a group, the position of an animal in a hierarchy, or its ability to defend resources, is often dependent upon body size and physical strength [63]. Age, size, condition, rank, relatedness, and experience can also influence the tendency to care for offspring and to participate in cooperative activities [64,65]. These and other cooperative and antagonistic behaviors can be partly regulated by mechanisms such as androgens [66], allostatic load [67], or receptor densities in specific brain regions [68]. In all cases, the underlying neural mechanisms regulating these behaviors remain largely unknown.

\section{Internal attributes: neural, neuroendocrine, and genetic mechanisms}

Behavioral neuroscientists have identified numerous endocrine and neural mechanisms that control behavioral decision-making and hence influence life-history traits, particularly in social species (Figure 1A). For example, specific neural circuits such as the dopaminergic reward system [69] and the brain social behavior network [70,71] regulate social behavior. Homologs of the nodes of this social decision-making network have been inferred across vertebrates, suggesting that this system is highly conserved [72,73]. In general, even subtle temporal, individual, or species-level differences in neural and molecular characteristics can result in functional variation in sensory, memory, valuation and motor centers, thereby contributing to the behavioral diversity we observe in nature. For example, neuroanatomical differences in the volume of a particular brain region can be related to the relative behavioral demands on that region [74-76]. At the cellular level, the release of neurochemicals such as biogenic amines [43] and neuropeptides [62] into specific brain regions can directly result in specific behavioral profiles. Although the expression patterns of neurochemical genes, particularly those encoding receptors, are remarkably conserved [77], quantitative variation in receptor density and/or levels of neurochemicals in specific network nodes is strongly associated with diversity in social behavioral attributes between individuals and across species $[68,78]$. Given the development of high-throughput sequencing technologies, it is now possible to quantify many of these neural and molecular characteristics in emerging model systems (e.g., [79-83]).

\section{Integrating across temporal and taxonomic scales}

Evolutionary processes influence all internal attributes in relation to a variety of external parameters. To explain these evolutionary processes it is important to obtain comparative quantitative data from a range of species and under multiple physiological and environmental conditions [84]. Once crucial external and internal attributes have been measured for numerous individuals and species, their inter-relationships can be identified via pairwise or multivariate statistical analyses [85]. By employing such an approach, we expect to discover functional relationships among individual attributes. For example, circulating hormone levels and receptor densities are functionally linked, and variation in these dimensions can be thought of as clustering into principal components reflecting functional units. At the same time, these mechanistic components likely also correspond to external attributes. For example, individual attributes such as sex, reproductive state, age, condition, and experience are likely to impinge upon the decision-making circuits via neuroendocrine and neuromodulator pathways [1]. The identification of co-variance patterns within and between axes representing mechanisms and those representing functional significance will likely reveal robust and representative measures of causal mechanisms associated with behavioral variation. Unfortunately, few if any such empirical studies have been conducted, despite that fact that we now have the analytical means to do so [86].

Behavioral, ecological, and neurobiological data from the same species are required to conduct this type of integrative analysis. At present, however, the most 
detailed ecological datasets often lack complementary neural measures and, conversely, ecological information is often lacking for (laboratory) species whose neural substrates have been studied in detail. In fact, it appears that, for established laboratory species as well as for emerging model systems, the amount of information available on ecology and/or reproductive biology might be inversely correlated with the extent of neurobiological information. Currently, most analyses of neural features of emerging model organisms involve pairwise comparisons of species or populations, with only a few instances of data collection in a broader phylogenetic context $[75,77,87]$. Those comparisons that exist are typically limited to only a few measures such as gross neuroanatomy [88], circulating hormone levels [89], and gene expression for small sets of loci [77]. Although such relatively limited comparisons can provide insights into evolutionary processes, more extensive species sampling and additional fine-grained neural and molecular measures are necessary to gain a full understanding of the evolution of these mechanisms and the behavior patterns they regulate.

\section{Revisiting Tinbergen's vision}

Fifty years after Tinbergen defined his proverbial 'four questions' [2], there is a tremendous opportunity for integrative studies on the ultimate and proximate mechanisms of complex behaviors such as sociality [7,9-11,16,90]. Although the number of animal species considered to be accepted as biomedical model systems is decreasing [91], this trend is both paradoxical and misguided given that the very notion of a model system is undergoing rapid change and might soon be obsolete [92], at last liberating us to (again) use the species that are best suited for the problem in question (i.e., Krogh's Principle [93]). Innovative research programs in diverse species are now possible thanks to advances in behavioral ecology, genomics, and neuroscience together with numerous technological breakthroughs that facilitate the collection of ever-larger and more detailed datasets than were imaginable even a few years ago. Systematic efforts are now needed to fill the gaps in our understanding of social behavior for species that are not the established biomedical model systems discussed here and elsewhere. The development of new model systems that create comprehensive behavioral, ecological, and neural datasets within the framework we have provided here will help us to fulfill Tinbergen's vision to understand truly the evolution of neuroethological mechanisms across all levels of biological organization and at all levels of analysis.

\section{Acknowledgments}

We thank the National Evolutionary Synthesis Center (NESCent) [US National Science Foundation (NSF) EF-0905606] for supporting our working group; Jim Goodson, and Emilia Martins for stimulating discussions; and Rayna Harris for assistance with manuscript preparation. H.A.H. is supported by NSF grant IOS-0843712; A.K.B. is supported by NSF IOS-1257162; D.T.B. is supported by NSF DEB-1119660; I.D.C. is supported by NSF PHY-0848755, NSF EAGER-1251424, NSF CNH1211972, Office of Naval Research N00014-09-1-1074, Army Research Office W911NG-11-1-0385 and Human Frontier Science Program award RGP0065/2012; R.L.E. is supported by NSF IOS-1051682 and NSF IOS1311347; L.D.H. is supported by NSF IIA-0853719 and NSF IIA0901056;P.L.H. is supported by the Natural Sciences and Engineering Research Council of Canada (NSERC) RGPIN 249685; M.T. is supported by Swiss National Science Foundation grant 310030B 138660; L.J.Y. is supported by US National Institutes of Health (NIH) grants MH064692 and P51OD11132; D.R.R. is supported by NSF IOS-1121435 and IOS-1257530

\section{References}

1 Taborsky, B. and Oliveira, R.F. (2012) Social competence: an evolutionary approach. Trends Ecol. Evol. 27, 679688

2 Tinbergen, N. (1963) On aims and methods of ethology. Z. Tierpsychol. 20, 410433

3 Sherman, P.W. (1988) The levels of analysis. Anim. Behav. 36, 616 619

4 Crews, D. and Moore, M. (1986) Evolution of mechanisms controlling mating behavior. Science 231, 121125

5 McNamara, J.M. and Houston, A.I. (2009) Integrating function and mechanism. Trends Ecol. Evol. 24, 670675

6 Blumstein, D.T. et al. (2010) Toward an integrative understanding of social behavior: new models and new opportunities. Front. Behav. Neurosci. 4, 34

7 O'Connell, L.A. and Hofmann, H.A. (2011) Genes, hormones, and circuits: an integrative approach to study the evolution of social behavior. Front. Neuroendocrinol. 32, 320335

8 Barrett, L. et al. (2013) Taking note of Tinbergen, or: the promise of a biology of behaviour. Philos. Trans. R. Soc. Lond. B: Biol. Sci. 368, 20120352

9 Oliveira, R.F. et al., eds (2008) Alternative Reproductive Tactics: An Integrative Approach, Oxford

10 MacDougall-Shackleton, S.A. (2011) The levels of analysis revisited. Philos. Trans. R. Soc. Lond. B: Biol. Sci. 366, 20762085

11 Ophir, A.G. (2011) Towards meeting Tinbergen's challenge. Horm. Behav. 60, 2227

12 Hitzemann, R. et al. (2013) Genes, behavior and next-generation RNA sequencing. Genes Brain Behav. 12, 112

13 Pennell, M.W. and Harmon, L.J. (2013) An integrative view of phylogenetic comparative methods: connections to population genetics, community ecology, and paleobiology. Ann. N. Y. Acad. Sci. 1289, 90105

14 Houle, D. (2010) Colloquium papers: Numbering the hairs on our heads: the shared challenge and promise of phenomics. Proc. Natl. Acad. Sci. U.S.A. 107 (Suppl.), 17931799

15 Wilson, A.J. et al. (2010) An ecologist's guide to the animal model. J. Anim. Ecol. 79, 1326

16 Healy, S.D. et al. (2009) Explanations for variation in cognitive ability: behavioural ecology meets comparative cognition. Behav. Processes 80,288294

17 Candolin, U. and Wong, B.B.M. (2012) Behavioural Responses to a Changing World: Mechanisms and Consequences, Oxford University Press

18 Robinson, G.E. et al. (2010) Empowering 21st century biology BioScience 60, 923930

19 Alcock, J. (2013) Animal Behavior: An Evolutionary Approach, Sinauer Associates

20 Dugatkin, L.A. (2013) Principles of Animal Behavior. (3rd edn), W.W Norton

21 Goodenough, J. et al. (2009) Perspectives on Animal Behavior, Wiley

22 Kappeler, P.M. et al. (2013) Constraints and flexibility in mammalian social behaviour: introduction and synthesis. Philos. Trans. R. Soc. Lond. B: Biol. Sci. 368, 20120337

23 Grafen, A. (1984) Natural selection, kin selection and group selection. In Behavioral Ecology (2nd edn) (Krebs, J.R. and Davies, N.B., eds), pp. 62 84, Blackwell Scientific

24 Springer, S.A. et al. (2011) Beyond the phenotypic gambit: molecular behavioural ecology and the evolution of genetic architecture. Mol. Ecol. 20, 22402257

25 Sokolowski, M.B. (2010) Social interactions in 'simple' model systems Neuron 65, 780794

26 Bolker, J. (2012) Model organisms: there's more to life than rats and flies. Nature 491, 3133

27 Rubenstein, D.R. (2007) Stress hormones and sociality: integrating social and environmental stressors. Proc. Biol. Sci. 274, 967975

28 Stacey, P.B. and Koenig, W.D. (1990) Cooperative Breeding in Birds: Long Term Studies of Ecology and Behaviour, Cambridge University Press 
29 Solomon, N.G. and French, J.A. (1997) Cooperative Breeding in Mammals, Cambridge University Press

30 Young, L.J. and Wang, Z. (2004) The neurobiology of pair bonding. Nat. Neurosci. 7, 10481054

31 Harris, R.M. and Hofmann, H.A. (2014) Neurogenomics of behavioral plasticity. Adv. Exp. Med. Biol. 781, 149168

32 Robinson, G.E. et al. (2008) Genes and social behavior. Science 322, 896900

33 Bourke, A.F.G. (2011) Principles of Social Evolution (Oxford Series in Ecology and Evolution), Oxford University Press

34 O'Connell, L.A. et al. (2012) Isotocin regulates paternal care in a monogamous cichlid fish. Horm. Behav. 61, 725733

35 Soma, K.K. et al. (2008) Novel mechanisms for neuroendocrine regulation of aggression. Front. Neuroendocrinol. 29, 476489

36 Toth, A.L. and Robinson, G.E. (2009) Evo-devo and the evolution of social behavior: brain gene expression analyses in social insects. Cold Spring Harb. Symp. Quant. Biol. 74, 419426

37 Shubin, N. et al. (2009) Deep homology and the origins of evolutionary novelty. Nature 457, 818823

38 Turner, L.M. et al. (2010) Monogamy evolves through multiple mechanisms: evidence from V1aR in deer mice. Mol. Biol. Evol. 27, 12691278

39 True, J.R. and Haag, E.S. (2001) Developmental system drift and flexibility in evolutionary trajectories. Evol. Dev. 3, 109119

40 McGary, K.L. et al. (2010) Systematic discovery of nonobvious human disease models through orthologous phenotypes. Proc. Natl. Acad. Sci. U.S.A. 107, 65446549

41 Oldfield, R.G. and Hofmann, H.A. (2011) Neuropeptide regulation of social behavior in a monogamous cichlid fish. Physiol. Behav. 102, 296303

42 Edwards, D.H. and Kravitz, E.A. (1997) Serotonin, social status and aggression. Curr. Opin. Neurobiol. 7, 812819

43 Miczek, K.A. et al. (2007) Neurobiology of escalated aggression and violence. J. Neurosci. 27, 1180321186

44 Nelson, R.J. and Trainor, B.C. (2007) Neural mechanisms of aggression. Nat. Rev. Neurosci. 8, 536546

45 Piprek, R.P. (2009) Genetic mechansisms underlying male sex determination in mammals. J. Appl. Genet. 50, 347360

46 Godwin, J. (2010) Neuroendocrinology of sexual plasticity in teleost fishes. Front. Neuroendocrinol. 31, 203216

47 Rhen, T. and Schroeder, A. (2010) Molecular mechanisms of sex determination in reptiles. Sex Dev. 4, 1628

48 Garrison, J.L. et al. (2012) Oxytocin/vasopressin-related peptides have an ancient role in reproductive behavior. Science 338, 540543

49 Wagenaar, D.A. et al. (2010) A hormone-activated central pattern generator for courtship. Curr. Biol. 20, 487495

50 Bergmüller, R. and Taborsky, M. (2010) Animal personality due to social niche specialisation. Trends Ecol. Evol. 25, 504511

51 Lott, D.F. (1991) Intraspecific Variation in the Social Systems of Wild Vertebrates, (Cambridge Studies in Behavioural Biology), Cambridge University Press

52 Pryke, S.R. et al. (2007) Frequency-dependent physiological trade-offs between competing colour morphs. Biol. Lett. 3, 494497

53 Dijkstra, P.D. et al. (2011) The role of physiology in the divergence of two incipient cichlid species. J. Evol. Biol. 24, 26392652

54 Sinervo, B. and Svensson, E. (2002) Correlational selection and the evolution of genomic architecture. Heredity (Edinb) 89, 329338

55 Zera, A.J. et al. (2007) Hormones in the field: evolutionary endocrinology of juvenile hormone and ecdysteroids in field populations of the wing-dimorphic cricket Gryllus firmus. Physiol. Biochem. Zool. 80, 592606

56 McGlothlin, J.W. and Ketterson, E.D. (2008) Hormone-mediated suites as adaptations and evolutionary constraints. Philos. Trans. R. Soc. Lond. B: Biol. Sci. 363, 16111620

57 Oldfield, R.G. et al. (2013) Arginine vasotocin and androgen pathways are associated with mating system variation in North American cichlid fishes. Horm. Behav. 64, 4452

58 Emlen, S.T. and Oring, L.W. (1977) Ecology, sexual selection, and the evolution of mating systems. Science 197, 215223

59 Koenig, W.D. and Pitelka, F.A. (1981) Ecological factors and kin selection in the evolution of cooperative breeding in birds. In Natural Selection and Social Behavior: Recent Research and New Theory (Alexander, R.D. and Tinkle, D.W., eds), pp. 261 280, Chiron
60 Komdeur, J. (1992) Importance of habitat saturation and territory quality for evolution of cooperative breeding in the Seychelles warbler. Nature 358, 493495

61 Lucia, K.E. et al. (2008) Philopatry in prairie voles: an evaluation of the habitat saturation hypothesis. Behav. Ecol. 19, 774783

62 Goodson, J.L. et al. (2012) Evolving nonapeptide mechanisms of gregariousness and social diversity in birds. Horm. Behav. 61, 239250

63 Hamilton, I.M. et al. (2005) Size differences within a dominance hierarchy influence conflict and help in a cooperatively breeding cichlid. Behaviour 142, 15911613

64 Clutton-Brock, T. (2002) Breeding together: kin selection and mutualism in cooperative vertebrates. Science 296, 6972

65 Bruintjes, R. and Taborsky, M. (2011) Size-dependent task specialization in a cooperative cichlid in response to experimental variation of demand. Anim. Behav. 81, 387394

66 Bender, N. et al. (2006) The relationship between social status, behaviour, growth and steroids in male helpers and breeders of a cooperatively breeding cichlid. Horm. Behav. 50, 173182

67 Rubenstein, D.R. (2009) Shen S-F. Reproductive conflict and the costs of social status in cooperatively breeding vertebrates. Am. Nat. 173, 650661

68 Lim, M.M. et al. (2005) Species and sex differences in brain distribution of corticotropin-releasing factor receptor subtypes 1 and 2 in monogamous and promiscuous vole species. J. Comp. Neurol. 487, 7592

69 Wise, R.A. (2005) Forebrain substrates of reward and motivation J. Comp. Neurol. 493, 115121

70 Newman, S.W. (1999) The medial extended amygdala in male reproductive behavior. A node in the mammalian social behavior network. Ann. N. Y. Acad. Sci. 877, 242257

71 Goodson, J.L. (2005) The vertebrate social behavior network: evolutionary themes and variations. Horm. Behav. 48, 1122

72 O'Connell, L.A. and Hofmann, H.A. (2011) The vertebrate mesolimbic reward system and social behavior network: a comparative synthesis. J. Comp. Neurol. 519, 35993639

73 Goodson, J.L. and Kingsbury, M.A. (2013) What's in a name? Considerations of homologies and nomenclature for vertebrate social behavior networks. Horm. Behav. 64, 103112

74 Healy, S.D. et al. (2005) The hippocampus, spatial memory and food hoarding: a puzzle revisited. Trends Ecol. Evol. 20, 1722

75 Pollen, A.A. et al. (2007) Environmental complexity and social organization sculpt the brain in Lake Tanganyikan cichlid fish. Brain Behav. Evol. 70, 2139

76 Lefebvre, L. and Sol, D. (2008) Brains, lifestyles and cognition: are there general trends? Brain Behav. Evol. 72, 135144

77 O'Connell, L.A. and Hofmann, H.A. (2012) Evolution of a vertebrate social decision-making network. Science 336, 11541157

78 Anacker, A.M.J. and Beery, A.K. (2013) Life in Groups: the roles of oxytocin in mammalian sociality. Fron. Behav. Neurosci. 7, 185

79 Whitfield, C.W. et al. (2003) Gene expression profiles in the brain predict behavior in individual honey bees. Science 302 , 296299

80 Toth, A.L. et al. (2007) Wasp gene expression supports an evolutionary link between maternal behavior and eusociality. Science 318, 441444

81 Cummings, M.E. et al. (2008) Sexual and social stimuli elicit rapid and contrasting genomic responses. Proc. Biol. Sci. 275, 393402

82 Renn, S.C. et al. (2008) Fish and chips: functional genomics of social plasticity in an African cichlid fish. J. Exp. Biol. 211, 30413056

83 Mukai, M. et al. (2009) Seasonal differences of gene expression profiles in song sparrow (Melospiza melodia) hypothalamus in relation to territorial aggression. PLoS ONE 4, 14

84 Pollen, A.A. and Hofmann, H.A. (2008) Beyond neuroanatomy: novel approaches to studying brain evolution. Brain Behav. Evol. 72, 145 158

85 O'Connell, L.A. and Hofmann, H.A. (2012) Social status predicts how sex steroid receptors regulate complex behavior across levels of biological organization. Endocrinology 153, 13411351

86 Rittschof, C.C. and Robinson, G.E. (2014) Genomics: moving behavioural ecology beyond the phenotypic gambit. Anim. Behav. 92,263270 
87 Goodson, J.L. and Kabelik, D. (2009) Dynamic limbic networks and social diversity in vertebrates: from neural context to neuromodulatory patterning. Front. Neuroendocrinol. 30, 429441

88 Striedter, G.F. (2005) Principles of Brain Evolution, Sinauer Associates

89 Hirschenhauser, K. et al. (2003) Comparative analysis of male androgen responsiveness to social environment in birds: the effects of mating system and paternal incubation. Horm. Behav. 43, 508519

90 Hayes, L.D. et al. (2011) Towards an integrative model of sociality in caviomorph rodents. J. Mammal. 92, 6577

91 Beery, A.K. and Zucker, I. (2011) Sex bias in neuroscience and biomedical research. Neurosci. Biobehav. Rev. 35, 565572

92 Losos, J.B. et al. (2013) Evolutionary biology for the 21st century. PLoS Biol. 11, e1001466

93 Krogh, A. (1929) The progress of physiology. Science 70, 200204

94 Belthoff, J. and Dufty, A.M. (1998) Jr Corticosterone, body condition and locomotor activity: a model for dispersal in screech-owls. Anim. Behav. 55, 405415
95 Nunes, S. et al. (1998) Body fat and time of year interact to mediate dispersal behaviour in ground squirrels. Anim. Behav. 55, 605614

96 Meylan, S. et al. (2002) Stress and body condition as prenatal and postnatal determinants of dispersal in the common lizard (Lacerta vivipara). Horm. Behav. 42, 319326

97 Keverne, E.B. and Curley, J.P. (2004) Vasopressin, oxytocin and social behaviour. Curr. Opin. Neurobiol. 14, 777783

98 Cushing, B.S. and Wynne-Edwards, K.E. (2006) Estrogen receptoralpha distribution in male rodents is associated with social organization. J. Comp. Neurol. 494, 595605

99 Ophir, A.G. et al. (2008) Variation in neural V1aR predicts sexual fidelity and space use among male prairie voles in semi-natural settings. Proc. Natl. Acad. Sci. U.S.A. 105, 12491254

100 Mabry, K.E. et al. (2011) Avpr1a length polymorphism is not associated with either social or genetic monogamy in free-living prairie voles. Anim. Behav. 81, 1118

101 Bateson, P. and Laland, K.N. (2013) Tinbergen's four questions: an appreciation and an update. Trends Ecol. Evol. 28, 712718 